\title{
Venous Manometry as an Adjunct for Diagnosis and Multimodal Management of Intracranial Hypertension due to Meningioma Compressing Sigmoid Sinus
}

\author{
Cletus Cheyuo, Charles L. Rosen, Ansaar Rai, Christopher P. Cifarelli, Rabia Qaiser
}

1.

Corresponding author: Rabia Qaiser, rabiahq@gmail.com

\section{Abstract}

Intracranial venous hypertension is a rare presentation of meningiomas in the transversesigmoid sinus region. We describe a case of a young patient presenting with intracranial hypertension due to a meningioma causing compression of the dominant sigmoid sinus. We were able to document the cerebral venous pressure gradient across the lesion confirming our hypothesis that compression of the sigmoid sinus from the meningioma was the cause of intracranial hypertension.

The patient is a 17-year-old male who presented with intracranial hypertension due to meningioma at the right dominant sigmoid sinus, which was treated by a Simpson grade IV surgical resection followed by stereotactic radiosurgery. Following treatment, his papilledema resolved and he remains symptom-free at 18 months.

In conclusion, venous manometry is a useful adjunct to diagnose intracranial hypertension in non-idiopathic causes of intracranial hypertension. A multimodal management approach of intracranial hypertension due to outflow obstruction from the dominant sinus led to an excellent recovery on follow up.

Categories: Radiology, Neurosurgery, Oncology

Keywords: intracranial hypertension, sigmoid sinus meningioma, venous manometry, stereotactic radiosurgey

\section{Introduction}

Received 05/09/2019

Review began 06/03/2019 Review ended 06/04/2019 Published 06/20/2019

\section{(C) Copyright 2019}

Cheyuo et al. This is an open access article distributed under the terms of the Creative Commons Attribution License CC-BY 3.0., which permits unrestricted use, distribution, and reproduction in any medium, provided the original author and source are credited.
Meningiomas are benign neoplasms that arise from arachnoid cap cells. The arachnoid cap cells protrude into the venous sinuses and are most abundant around the superior sagittal sinus. The posterior fossa is the site for $7-10 \%$ meningiomas, of which $10 \%$ involve the transverse or sigmoid sinus [1]. Meningiomas are commonly asymptomatic but can present with symptoms of mass effect such as headache as well as symptoms peculiar to location. Invasion of the venous sinuses by a meningioma can cause obstruction of the venous drainage leading to increases in the intracranial pressure, and venous infarctions [2].

A rare syndrome of intracranial hypertension has been described in a few meningiomas with venous sinus invasion [2-3]. Intracranial hypertension is described as intracranial pressure greater than $20 \mathrm{~cm} \mathrm{H2O}$, which manifests clinically as headaches, nausea and vomiting as well as visual symptoms such as diplopia and vision loss over a long period of time. Diagnosis is 


\section{Cureus}

based on clinical presentation, ophthalmological evaluation for papilledema and radiological demonstration of meningioma obstructing the sinus. Venous manometry has been used previously for diagnosing idiopathic intracranial hypertension causing headaches [4]. However, to the best of our knowledge this is the first time it has been utilized for measuring the cerebral venous pressure gradient across a lesion. We describe the case of a 17-year-old male who presented with intracranial hypertension due to a meningioma at the right dominant sigmoid sinus, which was treated by surgical debulking followed by stereotactic radiosurgery.

\section{Case Presentation}

The patient is a 17-year-old male who presented with 3-month history of headache, worse in the morning and associated with blurry vision, nausea and vomiting. He initially presented to an optometrist at an outside hospital, who detected bilateral papilledema and referred him for further workup. On clinical examination, he was neurologically intact. Magnetic resonance imaging (MRI) of the brain, with gadolinium contrast, showed an enhancing $1.5 \mathrm{~cm}$ extra-axial mass causing stenosis of the dominant right sigmoid sinus (Figure 1).

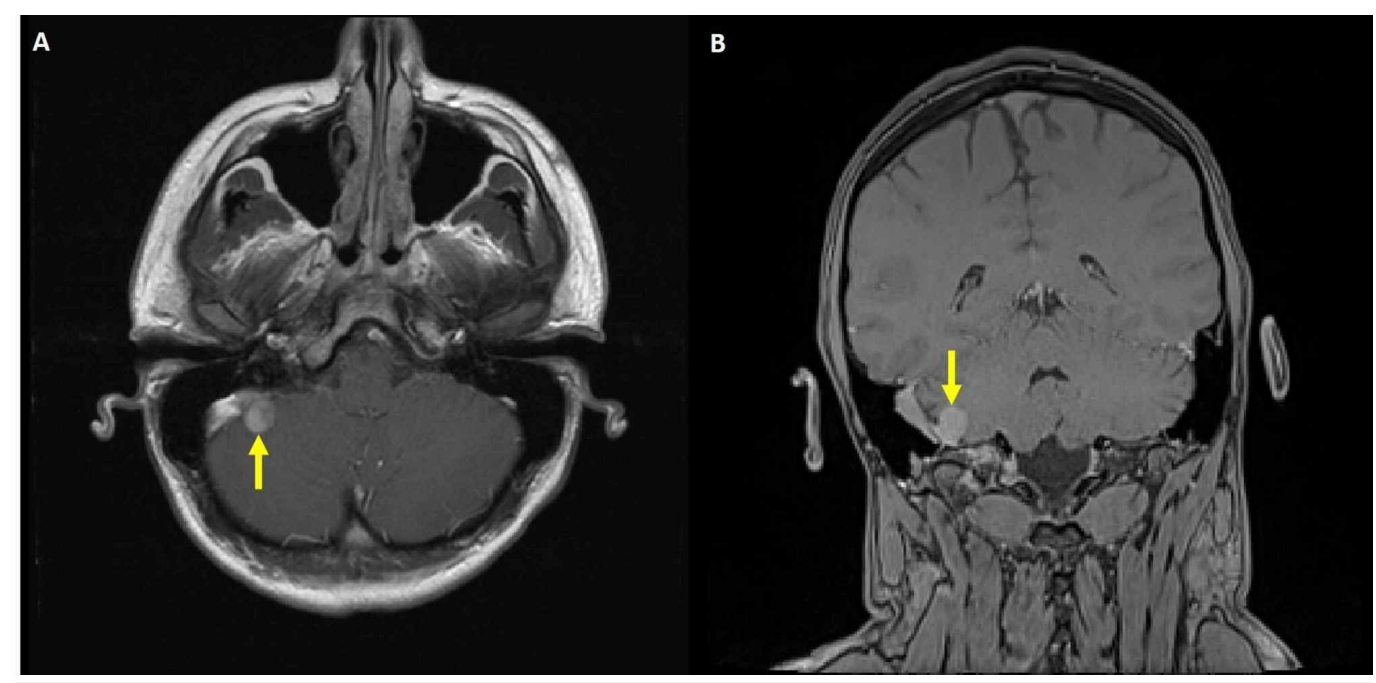

FIGURE 1: Preoperative magnetic resonance imaging (MRI) brain with gadolinium contrast

(A) Axial and (B) coronal views showing an enhancing $1.5 \mathrm{~cm}$ extra-axial mass (solid yellow arrows) causing stenosis of the right sigmoid sinus.

MRI of the orbits, with gadolinium contrast, showed the classic features of papilledema [5]; including flattening of the posterior sclera, enhancement of the prelaminar optic nerve, distension of the peri-optic subarachnoid space, intraocular protrusion of the prelaminar optic nerve and vertical tortuosity of the orbital optic nerve (Figure 2). 


\section{Cureus}

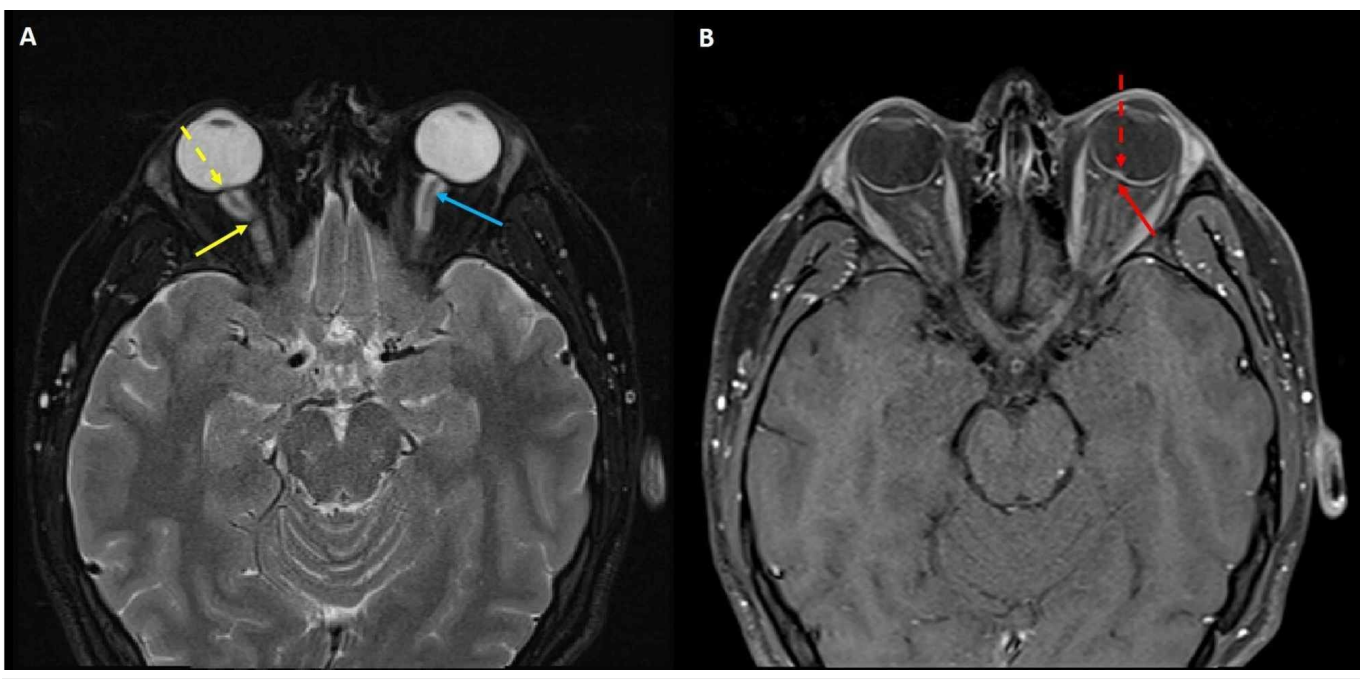

FIGURE 2: Magnetic resonance imaging (MRI) of the orbits with gadolinium contrast demonstrating papilledema

(A) Axial T2W showing flattening of the posterior sclera (dashed yellow arrow), distension of the peri-optic subarachnoid space (solid blue arrow) and vertical tortuosity of the orbital optic nerve (solid yellow arrow) and (B) Axial T1W with contrast showing enhancement of the prelaminar optic nerve (solid red arrow) and intraocular protrusion of the prelaminar optic nerve (dashed red arrow).

The patient also underwent lumbar puncture with fluoroscopic guidance with an opening pressure of $45 \mathrm{mmHg}$. Diagnostic cerebral angiogram and venous manometry were also performed as follows: A single wall needle was utilized to access the right common femoral artery and using the Seldinger technique a 5-French sheath was placed and connected to continuous flush. Cerebral arteriography was performed with a 5-French catheter. Subsequently the right common femoral vein was accessed with a 5 -French vascular access sheath which was connected to a continuous flush. Using a guidewire, a chaperone 6French catheter was navigated to the level of the right internal jugular vein. Subsequently through the chaperone a Prowler catheter (Codman Neurovascular, New Brunswick, New Jersey, USA) was advanced over an Avigo guidewire (Medtronic, Minneapolis, Minnesota, USA) into the right superior sagittal sinus, right transverse sinus, right sigmoid sinus and right internal jugular vein with pressure measurements at each of those locations. Catheter and sheath were removed at the end of the procedure from both the right common femoral artery and vein. Findings included a filling defect within the right sigmoid sinus due to the mass lesion (Figure 3). 


\section{Cureus}

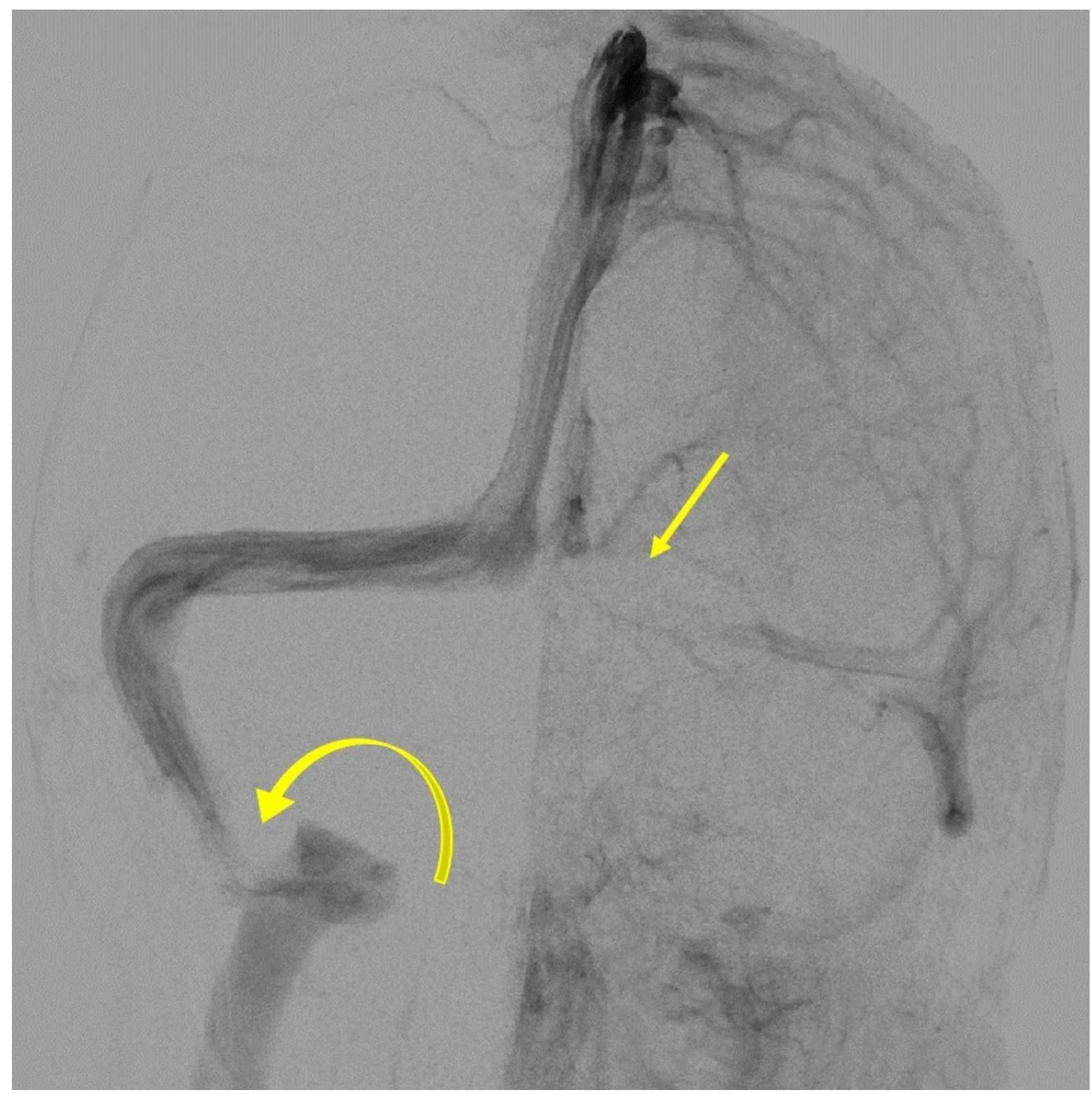

FIGURE 3: Diagnostic cerebral angiogram showing a filling defect within the right sigmoid sinus (curved yellow arrow) due to the mass lesion, and a hypoplastic left transverse sinus (straight yellow arrow).

There was no delay in transit of contrast from the right straight sinus/ torcula to the right internal jugular vein. Manometry showed a central venous pressure of $36 \mathrm{mmHg}$ (normal: 2-6 $\mathrm{mmHg}$ ) within the right transverse sinus and $10 \mathrm{mmHg}$ (normal: $2-6 \mathrm{~mm} \mathrm{Hg}$ ) within the right internal jugular vein (a $26 \mathrm{~mm} \mathrm{Hg}$ pressure gradient across the mass on the right sigmoid sinus). Based on the patient's clinical signs of increased intracranial pressure and the pressure gradient of $26 \mathrm{~mm} \mathrm{Hg}$ (normal: $<8 \mathrm{~mm} \mathrm{Hg}$ ) across the mass on the right sigmoid sinus, the decision was made to perform surgical debulking of the tumor followed by postoperative radiosurgery. Of note cerebral venous pressure gradient has been defined as $\geqslant 8 \mathrm{mmHg}$ on venographic manometry [4]. An external ventricular drain was placed to drain cerebrospinal fluid for brain relaxation during surgery. A right-sided stereotactic retrosigmoid craniotomy was then performed, with the patient in the three-quarter prone position. Frozen section histology was consistent with a meningioma. Simpson grade IV resection of the tumor was carried out as this was considered safest in order to prevent injury to the dominant sigmoid sinus. Postoperative MRI brain with gadolinium contrast showed the residual tumor (Figure 4). 


\section{Cureus}

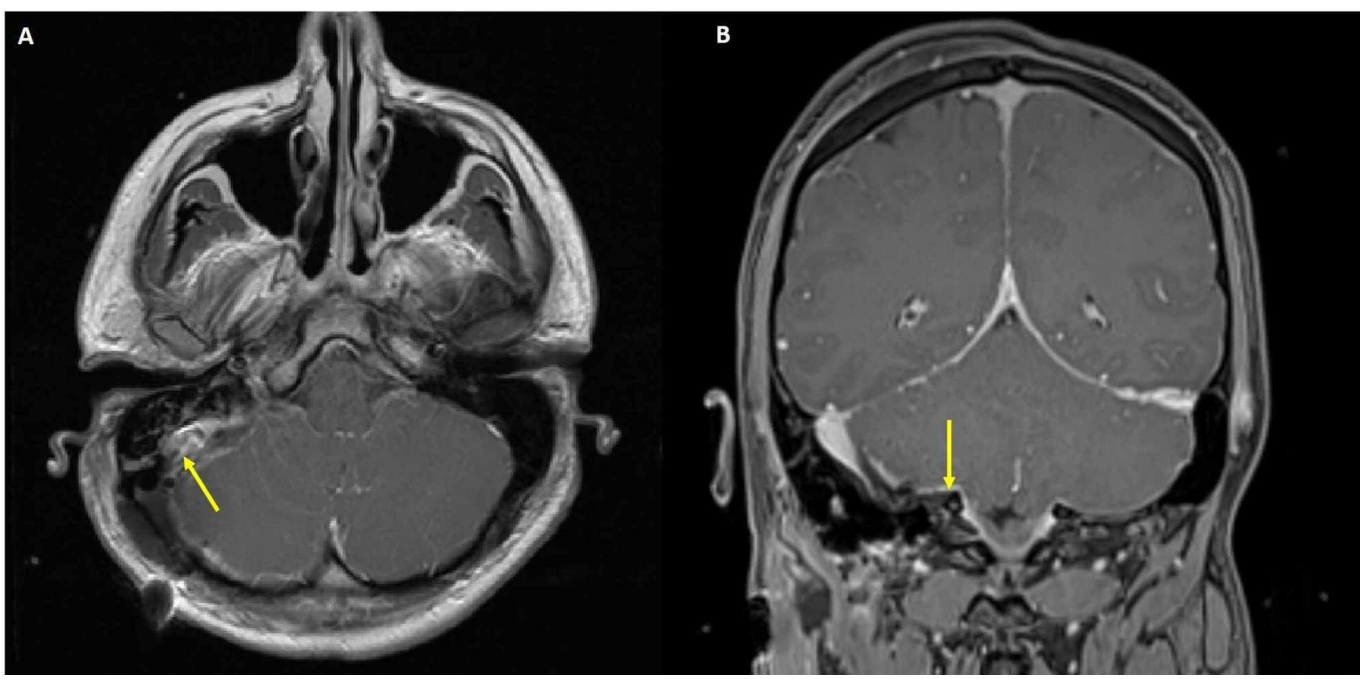

\section{FIGURE 4: Post-operative magnetic resonance imaging (MRI) brain with gadolinium contrast}

(A) Axial and (B) coronal views showing partial resection of tumor at the right sigmoid sinus region (solid yellow arrows)

The final pathology of the tumor was reported as WHO grade I meningioma. Given the high rate of meningioma re-growth after Simpson grade IV resection, we decided to treat the residual tumor with stereotactic radiosurgery. The patient underwent Gamma Knife Radiosurgery to the residual meningioma at six months postoperatively, consisting of $12.5 \mathrm{~Gy}$ to the $50 \%$ isodose line with a total number of four isocenters. At immediate follow up, patient's symptoms and papilledema had resolved. He remains asymptomatic at his 18-month follow-up.

\section{Discussion}

Posterior fossa meningiomas constitute $7-10 \%$ of all meningiomas, with $10 \%$ involving the transverse or sigmoid sinuses [1]. Majority of patients with meningiomas are asymptomatic. However, symptomatic patients often present with symptoms of mass effect such as headache as well as symptoms peculiar to location. Invasion of the venous sinuses by a meningioma can cause obstruction of venous drainage leading to increase in intracranial pressure and papilledema. In this report, we described the management of a 17-year-old male who presented with intracranial hypertension caused by a meningioma at the right dominant sigmoid sinus. To the best of our knowledge the cerebral venous pressure gradient has not been recorded in any such cases.

Intracranial hypertension is defined by a sustained increase in the intracranial pressure of more than $20 \mathrm{~cm} \mathrm{H2O} \mathrm{[6-7].} \mathrm{Intracranial} \mathrm{hypertension} \mathrm{caused} \mathrm{indirectly} \mathrm{by} \mathrm{a} \mathrm{mass} \mathrm{compressing} \mathrm{part}$ of the intracranial dural sinuses, resulting in obstruction of venous drainage, has been described [2-3]. Intracranial hypertension due to a mass compressing the dural sinuses should be distinguished from idiopathic intracranial hypertension which requires an increase in the intracranial pressure of more than $20 \mathrm{~cm} \mathrm{H2O}$, in the setting of normal ventricular size, absence of an intracranial mass and absence of neurological deficits [8]. The mechanism by which compression of the dural sinuses result in increased intracranial pressure is explained by the modified Kellie-Monroe doctrine, which states that the sum of intracranial volumes is constant, and that an increase in any component must be offset by an equal decrease in another component [9]. Cerebrospinal fluid is absorbed at the arachnoid granulations based on a pressure gradient [10]. In dural sinus compression, there is increase in venous sinus pressure 
proximal to the site of obstruction, resulting in reversal of the arachnoid granulation-venous sinus pressure gradient and impairment of cerebrospinal fluid absorption [1]. Venous congestion with impairment of the cerebrospinal fluid absorption results in intracranial hypertension.

The diagnosis of intracranial hypertension is based on signs and symptoms of increased intracranial pressure, brain imaging and objective demonstration of increased cerebrospinal fluid pressure. Our patient presented with classic clinical findings of chronic headache and papilledema. Papilledema was demonstrated on fundoscopy and also on brain imaging. Passi et al. described classic MRI findings in papilledema [5]. Our patient demonstrated all these findings, which included flattening of the posterior sclera, enhancement of the prelaminar optic nerve, distension of the peri-optic subarachnoid space, intraocular protrusion of the prelaminar optic nerve and vertical tortuosity of the orbital optic nerve. Lumbar puncture confirmed elevation of cerebrospinal fluid pressure. Venous manometry is a very useful quantitative adjunct in confirming venous obstruction and in planning of treatment options such as stenting [11]. It is especially important in cases where symptoms of intracranial hypertension are less obvious. Our patient underwent venography and manometry and was found to have a pressure gradient of $26 \mathrm{mmHg}$ across the mass on the right sigmoid sinus.

One of the goals of treatment for intracranial hypertension caused by a meningioma compressing the dural venous sinus is to relieve the obstruction. Options for achieving this include surgical resection, stereotactic radiosurgery, stenting or a combination of these modalities. The criteria for venous stenting include central cerebral venous pressure of $\geqslant 22 \mathrm{~mm}$ $\mathrm{Hg}$ and a pressure gradient of at least $8 \mathrm{mmHg}$. Venous stenting for intracranial hypertension is minimally invasive and is especially useful when the patient is considered high risk for open cranial surgery [11-13]. Even though our patient met the criteria for venous stenting, stenting was not offered because of the potential for re-stenosis from continued tumor growth and intimal hyperplasia. Another disadvantage of the option of stenting, especially in this pediatric patient, would be the need for long-term antiplatelet therapy to prevent stent thrombosis. Our patient was offered surgical debulking of the meningioma (Simpson grade IV) followed by stereotactic radiosurgery to the residual tumor.

Mazur et al. described the nuances of surgical management of meningiomas involving the transverse or sigmoid sinus [3]. During surgical planning it is important to determine the degree of intravascular tumor invasion, the degree of sinus occlusion and the anatomy of venous outflow. This is achieved with preoperative imaging modalities including magnetic resonance angiography, computed tomography (CT) angiography and cerebral angiography. Cerebral angiography is particularly important in delineating the venous anatomy in the case of a unilateral dominant transverse-sigmoid sinus involvement [14]. Cerebral angiography revealed that our patient had a filling defect within the right sigmoid sinus due to the mass lesion. There was no delay in transit of contrast from the right straight sinus/torcula to the right internal jugular vein. There was also no arterial supply from the intracranial circulation, but there was a small tortuous feeding vessel from the ascending pharyngeal artery. The left transverse sinus was hypoplastic. The occurrence of the tumor around a dominant right sigmoid sinus with partial occlusion partly informed our conservative approach to tumor resection. Care was taken during surgery to preserve bridging veins so as to avoid postoperative edema and venous infarction. The tumor was extravascularly debulked without opening the sinus. Inadvertent opening of the venous sinus is associated with increased risk of airembolism. In this case, injuring the dominant sinus would have been riskier given that even compression was resulting in intracranial hypertension.

Stereotactic radiosurgery for meningiomas at the transverse or sigmoid sinus is rarely reported, and this report is one of the few reported. Nowak et al. reported the surgical management of four jugular foramen meningiomas by transcondylar approach with sigmoid sinus ligation. One 
of the patients underwent stereotactic radiosurgery postoperatively for residual tumor [15]. Nicolato et al. also reported on their experience of gamma knife radiosurgery for 62 posterior fossa meningiomas but none of the meningiomas were at the sigmoid sinus [16]. Possible complications to radiosurgery could include edema from irradiation of peritumoral veins. However, excellent meningioma control has been reported with stereotactic radiosurgery [17]. Our patient had resolution of symptoms of intracranial hypertension including the papilledema and there has been no tumor regrowth during the admittedly short follow-up of 18 months.

\section{Conclusions}

Sigmoid sinus meningioma can cause intracranial hypertension. Conservative surgical resection followed by stereotactic radiosurgery offers immediate relief of symptoms and excellent tumor control while avoiding the morbidity of a more radical surgical approach. In addition, using venographic manometry provides confirmation of the pathophysiology of intracranial hypertension in this patient.

\section{Additional Information Disclosures}

Human subjects: Consent was obtained by all participants in this study. Conflicts of interest: In compliance with the ICMJE uniform disclosure form, all authors declare the following: Payment/services info: All authors have declared that no financial support was received from any organization for the submitted work. Financial relationships: All authors have declared that they have no financial relationships at present or within the previous three years with any organizations that might have an interest in the submitted work. Other relationships: All authors have declared that there are no other relationships or activities that could appear to have influenced the submitted work.

\section{References}

1. Chausson N, Bocquet J, Aveillan M, Olindo S, Signaté A, Merle H, Smadja D: Intracranial hypertension caused by a meningioma compressing the transverse sinus. J Clin Neurosci. 2010, 17:1589-1592. 10.1016/j.jocn.2010.03.039

2. Pillai A, Kumar S, Kumar A, Panikar D: An unusual parasagittal tumour with acute blindness and response to cerebrospinal fluid shunting. J Clin Neurosci. 2007, 14:1112-1116. 10.1016/j.jocn.2006.01.006

3. Mazur MD, Cutler A, Couldwell WT, Taussky P: Management of meningiomas involving the transverse or sigmoid sinus. Neurosurg Focus. 2013, 35:9. 10.3171/2013.8.FOCUS13340

4. Levitt MR, Hlubek RJ, Moon K, et al.: Incidence and predictors of dural venous sinus pressure gradient in idiopathic intracranial hypertension and non-idiopathic intracranial hypertension headache patients: results from 164 cerebral venograms. J Neurosurg. 2017, 126:347-353. 10.3171/2015.12.JNS152033

5. Passi N, Degnan AJ, Levy LM: MR imaging of papilledema and visual pathways: effects of increased intracranial pressure and pathophysiologic mechanisms. AJNR Am J Neuroradiol. 2013, 34:919-924. 10.3174/ajnr.A3022

6. Friedman DI, Jacobson DM: Diagnostic criteria for idiopathic intracranial hypertension. Neurology. 2002, 59:1492-1495. 10.1212/01.WNL.0000029570.69134.1B

7. Kim AW, Trobe JD: Syndrome simulating pseudotumor cerebri caused by partial transverse venous sinus obstruction in metastatic prostate cancer. Am J Ophthalmol. 2000, 129:254-256. 10.1016/S0002-9394(99)00326-8

8. Quan T, Li X, Xu H, Lin Y, Liu C, Li D, Guan S: Percutaneous endovascular biopsy in the diagnosis of venous sinus lesions: technical note. J Neurosurg. 2018, 1:5. 10.3171/2018.3.JNS173143

9. Mokri B: The Monro-Kellie hypothesis: applications in CSF volume depletion . Neurology. 2001, 56:1746-1748. 10.1212/WNL.56.12.1746 
10. Griffith HB, Jamjoom AB: The treatment of childhood hydrocephalus by choroid plexus coagulation and artificial cerebrospinal fluid perfusion. Br J Neurosurg. 1990, 4:95-100. 10.3109/02688699008992706

11. Teleb MS, Cziep ME, Lazzaro MA, Gheith A, Asif K, Remler B, Zaidat OO: Idiopathic intracranial hypertension: a systematic analysis of transverse sinus stenting. Interv Neurol. 2013, 2:132-143. 10.1159/000357503

12. Fargen KM, Spiotta AM, Hyer M, Lena J, Turner RD, Turk AS, Chaudry I: Comparison of venous sinus manometry gradients obtained while awake and under general anesthesia before venous sinus stenting. J Neurointerv Surg. 2017, 9:990-993. 10.1136/neurintsurg-2016-012608

13. Zilani G, Pereira EA, Baig F, Uberoi R, Kuker W, Kerr RS: Venoplasty and stenting of a jugular foramen meningioma. Br J Neurosurg. 2009, 23:557-560. 10.1080/02688690903254368

14. Hwang SK, Gwak HS, Paek SH, Kim DG, Jung HW: Guidelines for the ligation of the sigmoid or transverse sinus during large petroclival meningioma surgery. Skull Base. 2004, 14:21-28. $10.1055 /$ s-2004-821356

15. Nowak A, Dziedzic T, Czernicki T, Kunert P, Marchel A: Surgical treatment of jugular foramen meningiomas. Neurol Neurochir Pol. 2014, 48:391-396. 10.1016/j.pjnns.2014.09.008

16. Nicolato A, Foroni R, Pellegrino M, Ferraresi P, Alessandrini F, Gerosa M, Bricolo A: Gamma knife radiosurgery in meningiomas of the posterior fossa. Experience with 62 treated lesions. Minim Invasive Neurosurg. 2001, 44:211-217. 10.1055/s-2001-19934

17. Kondziolka D, Mathieu D, Lunsford LD, Martin JJ, Madhok R, Niranjan A, Flickinger JC: Radiosurgery as definitive management of intracranial meningiomas. Neurosurgery. 2008, 62:53-58. 10.1227/01.NEU.0000311061.72626.0D 